\title{
Comprehensive molecular cytogenetic investigation of chromosomal abnormalities in human medulloblastoma cell lines and xenograft ${ }^{1}$
}

\author{
Naji Aldosari, Rodney N. Wiltshire, Amalia Dutra, Evelin Schrock, Roger E. \\ McLendon, Henry S. Friedman, Darell D. Bigner, and Sandra H. Bigner ${ }^{2}$ \\ Departments of Pathology (N.A., R.N.W., R.E.M., D.D.B., S.H.B) and Surgery (H.S.F.), Duke University \\ Medical Center, Durham, NC 27710; Tumor Genetics and Molecular Cytogenetics, Institute for Molecular \\ Biotechnology, Beutenbergstrasse 11, 07745 Jena, Germany (E.S.); National Human Genome Research \\ Institute, National Institutes of Health, Bethesda, MD 20892 (A.D.)
}

Cell lines and xenografts derived from medulloblastomas are useful tools to investigate the chromosomal changes in these tumors. Here we used G-banding, fluorescence in situ hybridization (FISH), spectral karyotyping (SKY), and comparative genomic hybridization to study 4 medulloblastoma cell lines and 1 xenograft. Cell line D-425 Med had a relatively simple karyotype, with a terminal deletion of $10 \mathrm{q}$ and amplification of MYC in double-minutes (dmins). FISH demonstrated that an apparent isochromosome $(17 q)$ by routine karyotyping was actually an unbalanced translocation between 2 copies of chromosome 17. Cell line D-556 Med also had a simple near-diploid stemline with an unbalanced 1;13 translocation resulting in a gain of $1 \mathrm{q}$, an isochromosome $(17 \mathrm{q})$, and dmins. These findings were initially described using routine G-banded preparations, and FISH showed that the dmins were an amplification of MYC and the $i(17 q)$ was an isodicentric $17 \mathrm{q}$ chromosome. The other finding was confirmed by FISH, SKY, and comparative genomic

Received 13 July 2001, accepted 8 October 2001.

${ }^{1}$ Supported by grant CA-68119 from the National Cancer Institute, National Institutes of Health, Bethesda, Md.

${ }^{2}$ Address correspondence and reprint requests to Sandra $\mathrm{H}$. Bigner, Duke University Medical Center, Department of Pathology, P.O. Box 3712, Durham, NC 27710.

${ }^{3}$ Abbreviations used are as follows: $\mathrm{CGH}$, comparative genomic hybridization; dmin, double-minute chromosome; FISH, fluorescence in situ hybridization; SKY, spectral karyotyping; WCP, whole chromosome painting probe. hybridization. Cell lines D-721 Med and D-581 Med had complex karyotypic patterns that could be completely characterized only when FISH and SKY were used. Xenograft D-690 Med also had a complex pattern that FISH and SKY were helpful in completely elucidating. Interestingly, balanced reciprocal translocations were seen as well as complicated unbalanced translocations and marker chromosomes. Comparative genomic hybridization demonstrated only a deletion of $10 \mathrm{q} 22-10 \mathrm{q} 24$, supporting the idea that despite the complexity of the chromosomal rearrangements, minimal alterations in the overall chromosomal content had occurred. This study demonstrates that routine cytogenetic preparations are adequate to describe chromosomal abnormalities in occasional medulloblastoma samples, but a broader spectrum of molecular cytogenetic methods is required to completely analyze most of these tumor samples. NeuroOncology 4, 75-85, 2002 (Posted to Neuro-Oncology [serial online], Doc. 01-005, February 11, 2002. URL $<$ neuro-oncology.mc.duke.edu>)

$\mathrm{P}$ ermanent cultured cell lines and xenografts derived from human tumors are useful reagents for a variety of biological, molecular genetic, biochemical, functional, and therapeutic studies. They are particularly helpful in investigations of chromosomal rearrangements, deletion, and other cytogenetic alterations, providing an abundant supply of neoplastic cells for detailed analysis of these abnormalities. Characterization of the chromosomal deviations in the cultured and xenograft cells often provides clues to the underlying genetic defects possessed 
Table 1. Karyotypic nomenclature for medulloblastoma cell lines and xenograft

Cases

D-425

D-556

D-721

D-690

D-581
Karyotype nomenclature

46,XY, del(10)(q23),i(17)(q10),+dmins[cp5].ish amp (8)(q24.1)(MYC),

$\operatorname{del}(10)(q 23)(w c p 10+, p c p 10 q 26-), \operatorname{der}(17) t(17 ; 17)(p 12 ; q 11.2)(w c p 17+, D 17 Z 1+, p c p 17 p+)$,

$17 \mathrm{p} 11.2(\mathrm{D} 17 \mathrm{~S} 122 \times 2)$

$46, X X,+1, \operatorname{dic}(1 ; 13)(p 11.1 ; q 34), i(17)(q 10),+d \operatorname{mins}[c p 10]$.ish amp(8)(q24.1)(MYC),

$\operatorname{dic}(1 ; 13)(w c p 1+, D 1 Z 1+; w c p 13+), \operatorname{idic}(17)(p 11.2)(w c p 17+, D 17 Z 1+, p c p 17 p+, H E R-2 / N E U \times 2)$,

17p11.2(D17S122x1)

$44, X_{1}-Y_{,}+\operatorname{der}(1) t(1 ; ?)(p 34.1 ; ?), \operatorname{der}(3) t(3 ; ?)(p 10: ?),+\operatorname{der}(3) t(3 ; ?)(p 10: ?),+9[3]$,

$\operatorname{der}(11) t(3 ; 11)(q 12 ; p 15), \operatorname{dic}(16 ; 22)(q 12.1 ; p 11.1), \operatorname{add}(18)(p 11.3) \times 2,+\operatorname{der}(18) t(18 ; ?)(q 10 ; ?)$,

$\operatorname{dic}(1 ; 19)(p 11.1 ; q 13.4),-20,-22,-22[c p 11] . i s h+\operatorname{der}(1) t(1 ; 18)(p 34.3 p 11.2)$

(wcp1+,D1Z1+;wcp18+,D18Z1-),dic(3;22)(q11.1;p11.1)(wcp3+,D3Z1+;wcp22+),

$+\operatorname{dic}(3 ; 22)(q 11.1 ; p 11.1)(w c p 3+, D 3 Z 1+; w c p 22+), \operatorname{der}(11) t(3 ; 11)(q 13.2 ; p 15)(w c p 3+; w c p 11+)$,

$\operatorname{dic}(16 ; 22)(q 11.1 ; p 11.1)(w c p 16+; w c p 22+), 17 p 11.2(D 17 S 122 \times 2), \operatorname{add}(18)(w c p 18+)$,

$+\operatorname{der}(18) t(18 ; 20)(p 11.1 ; q 11)(w c p 18+; w c p 22+), \operatorname{dic}(1 ; 19)(w c p 1+, D 1 Z 1+; w c p 19+)$

$46, Y, \operatorname{der}(X) t(X ; 7)(p 11.2 ; p 13), \operatorname{der}(2) t(2 ; 4)(p 13 ; p 14) t(2 ; 11)(q 37 ; q 21), \operatorname{der}(4) t(4 ; 12)(p 14 ; q 12)$,

$\operatorname{der}(6) t(6 ; ?)(q 21 ; ?),-7, t(8 ; 15)(q 22 ; q 22)[2], \operatorname{der}(10) t(10 ; ?)(q 22 ; ?), \operatorname{del}(11)(q 21)$,

$\operatorname{der}(11) t(11 ; 12)(p 15 ; q 22), \operatorname{der}(12) t(12 ; ?)(q 13 ; ?),-12, \operatorname{ins}(13 ; ?)(q 21 ; ?), \operatorname{der}(22) t(7 ; 22)(q 21 ; q 13)$,

$+2 \operatorname{mar}[c p 7]$. ish $\operatorname{der}(X) t(X ; 7)(p 11.2 ; p 11.2)(w c p X+, D X Z 1+; w c p 7+, D 7 Z 1-)$,

$\operatorname{der}(2) \mathrm{t}(2 ; 9)(\mathrm{p} 15 ; \mathrm{q} 32) \mathrm{t}(2 ; 11)(\mathrm{q} 37.1 ; \mathrm{q} 21)(w c p 2+; w c p 9+; w c p 11+), t(4 ; 12)(\mathrm{p} 14 ; q 13)$

$(w c p 4+; w c p 12+), t(6 ; 20)(q 25 ; p 11)(w c p 6+; w c p 20+), \operatorname{der}(7) t(X ; 7)(p 11.2 ; p 11.2) t(2 ; 7)(p 13 ; q 11.2)$

$(w c p X+; w c p 7+, D 7 Z 1+; w c p 2+), t(8 ; 15)(w c p 8+; w c p 15+), \operatorname{der}(9) t(9 ; 22)(q 32 ; q 13.1)$

(wcp9+;wcp22+),del(10)(q22q24)(wcp10+,pcp10q26+),del(11)(q14)(wcp11+),

$\mathrm{t}(11 ; 12)(\mathrm{p} 13 ; q 13)(w c p 11+; w c p 12)$, ins $(13 ; 1)(q 14 ; q 25 q 31)(w c p 13+; w c p 1+)$,

17p11.2(D17S122×2), der(22)t(7;22)(q11.2;q13)(wcp7+;wcp22+)

$47, X,-Y, a d d(1)(q 44), \operatorname{der}(2) t(1 ; 2)(q 21 ; p 23),+\operatorname{der}(2) t(2 ; 3)(q 23 ; q 23),+\operatorname{der}(3) t(3: 3)(p 25 ; q 21)$,

$\operatorname{der}(5) t(2 ; 5)(q 24 ; p 15.1),+7, \operatorname{add}(9)(p 24), \operatorname{der}(10) t(10: ?)(q 22 ; ?) \operatorname{add}(10)(q 23.2), \operatorname{add}(13)(p 11)$,

-15, del(17)(p12),-22[3],+mar[cp10].ish $\operatorname{der}(1) t(1 ; 10)(q 42 ; q 24)(w c p 1+, D 1 Z 1+; w c p 10+, D 10 Z 1-)$

$\operatorname{der}(2) t(1 ; 2)(q 22 ; p 23)(w c p 1+; w c p 2+),+\operatorname{der}(2 ; 10) t(2 ; 10)(q 23 ; p 11.2) t(10 ; 13)(q 21 ; q 22)$

(wcp2+;wcp10+,D10Z1+;wcp13+),+der(3;15)(p24;q12)t(13;15)(q14;q26)

(wcp3+;wcp15+;wcp13+), der(5)t(4;5)(q23;p15.1)(wcp4+;wcp5+),+7(wcp7+),

$\operatorname{der}(9) \mathrm{t}(1 ; 9)(\mathrm{q} 42 ; \mathrm{p} 21)(w c p 1+; w c p 9+), \operatorname{der}(10) \mathrm{t}(2 ; 10)(\mathrm{q} 21 ; q 11.2) \mathrm{t}(2 ; 13)(q 21 ; q 22)$

(wcp2+;wcp10+,D10Z1+;wcp13+), der(10)t(9;10)(p21;q22)(wcp9+;wcp10+,D10Z1+),

$\operatorname{der}(13) t(13 ; 22)(p 11.2 ; q 11.2)(w c p 13+; w c p 22+),+\operatorname{del}(15)(q 12)(w c p 15+)$,

$\operatorname{del}(17)(p 11.2)(w c p 17+, p c p 17 p+), 17 p 11.2(D 17 S 122 \times 1)$ by the original cells from which they were derived. For example, translocations may be associated with specific genetic rearrangements; chromosomal deletions or losses may point to the locations of altered tumor suppressor genes; and $\mathrm{dmins}^{3}$ and homogeneously staining regions are generally associated with gene amplification.

Recent developments in molecular cytogenetics, such as FISH, SKY, and CGH, have provided powerful tools to aid classic G-banding analysis in characterizing complex chromosomal patterns. FISH, when WCPs are employed, is useful for confirming chromosomal rearrangements first identified by G-banding analysis and for elucidating chromosomal breakpoints (Gozzetti and Le Beau, 2000; Wiltshire et al., 2001). In addition, single-copy gene probes can determine the copy number status of a specific gene, as well as whether or not the gene is rearranged, without the need for metaphase spreads. However, FISH analysis can be cumbersome when examining multiple marker chromosomes. SKY analysis is an excellent correlate to FISH and classic karyotypic analyses (Schrock and Padilla-Nash, 2000). All normal and aberrant chromo- somes are identified with an array of distinct color combinations in one experiment. Similar to classic cytogenetics and WCP FISH analyses, the quality of metaphase spreads and the size of the altered chromosomal region can be limiting factors when discerning breakpoints. On the other hand, CGH is unique in molecular cytogenetics because metaphase chromosomes from the sample are not required. The DNA from all of the cells in the tumor is studied to get a net change in the copy number status of DNA sequences compared with the normal copy number complement. Deletions and low- and high-level gains of genetic material are detected in one analysis; however, balanced rearrangements are not readily discerned with CGH (Lichter et al., 2000). Separately, these techniques provide useful but sometimes incomplete information about chromosomal alterations. In combination, the data from these techniques can better elucidate the overall genetic composition of any sample under investigation.

In this study, the above methods were applied to 4 cell lines and 1 xenograft, which were derived from human medulloblastomas. We were able to completely charac- 
terize the complex karyotypic alterations in these cases and demonstrate subtle rearrangements that were not detectable by routine G-banded karyotypes. The results were consistent with reported cytogenetic analyses and provided more insight into the genetic complexity of these medulloblastoma specimens.

\section{Materials and Methods}

\section{Cytogenetic Procedure}

Chromosomal analysis was performed on 4 cell lines and a xenograft. D-425 was derived from a 5-year-old female (Bigner and Vogelstein, 1990), D-556 from a 7-year-old female, D-721 from a 2-year-old male, D-690 from a 16-year-old male (Bigner and Schrock, 1997), and D-581 from a 2-year-old male. Cells were arrested in metaphase with colcemid, treated with a hypotonic solution, and processed in fixative according to standard techniques. The slides were aged overnight at $60^{\circ} \mathrm{C}$, trypsinized, then stained with Wright's stain. Chromosomal analysis was performed by following routine cytogenetic procedures. Twenty metaphases were counted, and a minimum of 5 metaphases were karyotyped and described according to the International System for Human Cytogenetics (ISCN; Mitelman, 1995).

\section{FISH}

FISH was used to identify marker chromosomes and chromosomal rearrangements that were difficult to discern with conventional cytogenetics methods. All probes were used according to the manufacturers' instructions. WCPs for chromosomes 1 to 22 and sex chromosomes, and centromeric probes (DXZ1, D1Z1, D3Z1, D7Z1, D10Z1, D17Z1, D18Z1) (Vysis, Inc., Downer's Grove, Ill.), were used to study the various rearranged chromosomes. Partial chromosome painting probes for the $\mathrm{p}$ and $\mathrm{q}$ arms of chromosome 17 and chromosome 10q26 (American Laboratory Technologies, Inc., Arlington, Va.), along with a HER-2/NEU gene-specific probe (17q12) from Vysis, Inc., were used to examine aberrant alterations involving chromosomes 17 and 10. The Vysis LSI c-myc probe (8q24.1) was used to study MYCC gene amplification.

\section{CGH}

CGH was performed according to the manufacturer's instructions (Vysis, Inc.). Briefly, $1 \mu \mathrm{g}$ of DNA extracted from each of the 4 cell lines and the xenograft was directly labeled by nick translation with spectrum green dUTP, and the same amount of normal reference DNA was labeled with spectrum red dUTP. Both labeled DNA were then hybridized to a slide containing normal lymphocyte metaphase spreads. Twenty metaphases were captured on a fluorescent microscope equipped with epifluorescent filters and a CCD (charge-coupled device) camera. The captured images were analyzed using Vysis QUIPS imaging and CGH analysis software. Excluded chromosome regions and established threshold ratios were the same as previously described (Wiltshire et al., 2000).

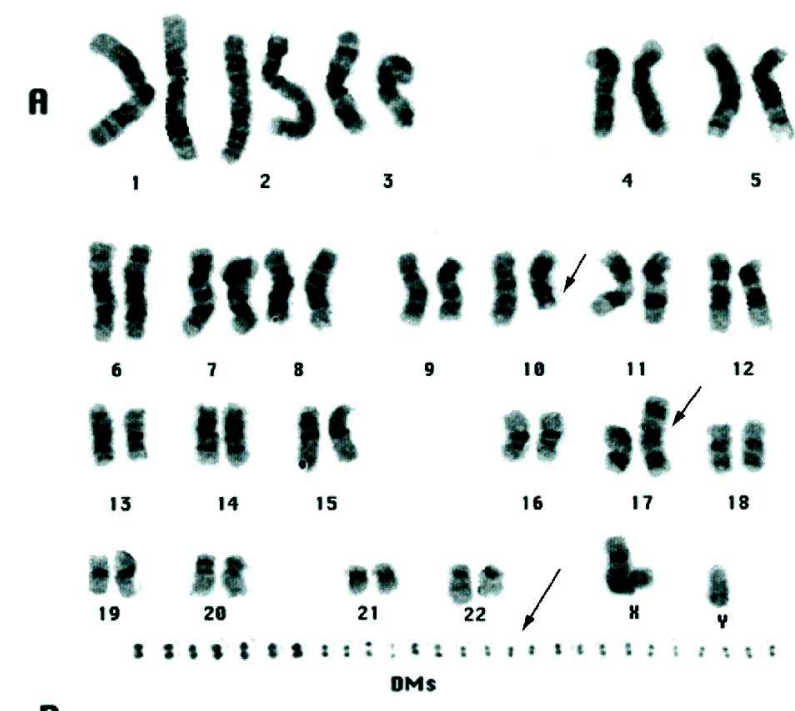

B

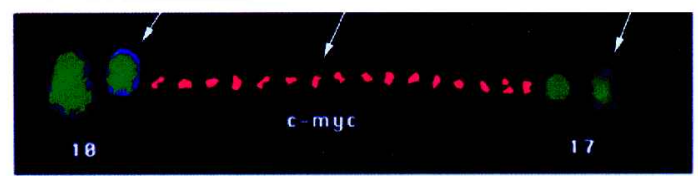

Fig. 1. Cell line D-425. A. Representative karyotype: $46, X Y$, del(10)(q23),i(17)(q10), +dmins. Arrows point to abnormal chromosomes 10 and 17 and to dmins. B. Composite panel showing results of FISH on normal and derivative chromosomes. Arrows indicate 10q deletion (WCP 10 SpectrumGreen), c-myc amplification in dmins (LSI c-myc SpectrumOrange), and derivative chromosome 17 (WCP 17 SpectrumGreen).

\section{SKY}

SKY uses the simultaneous labeling of painting probes to visualize each chromosome within a metaphase in a different color. A Fourier transform interferometer coupled to a CCD camera (Spectracube ${ }^{\mathrm{TM}}$, Applied Spectral Imaging, Carlsbad, Calif.) was used for image acquisition. A single image is captured and the complete emission spectra are measured for each image point. Chromosome classification is based on the unique spectral information characteristic for each chromosome and is performed using the SKY-View software (Applied Spectral Imaging) (Bigner and Schrock, 1997). 4'6-Diamidino-2-phenylindole banding was used to estimate chromosomal breakpoints.

\section{Results}

G-band karyotypic analysis revealed abnormal stemlines in all 4 cell lines and the xenograft. Two of the cell lines had few chromosomal alterations, and the other 3 had many complex chromosomal rearrangements. Table 1 shows the final karyotypic descriptions that resulted from analyses by G-banding and FISH.

\section{D-425}

This cell line had a diploid chromosome number and contained few chromosomal abnormalities that involve chromosomes 10 and 17 and dmins (Table 1 and Fig. 1). All 
A

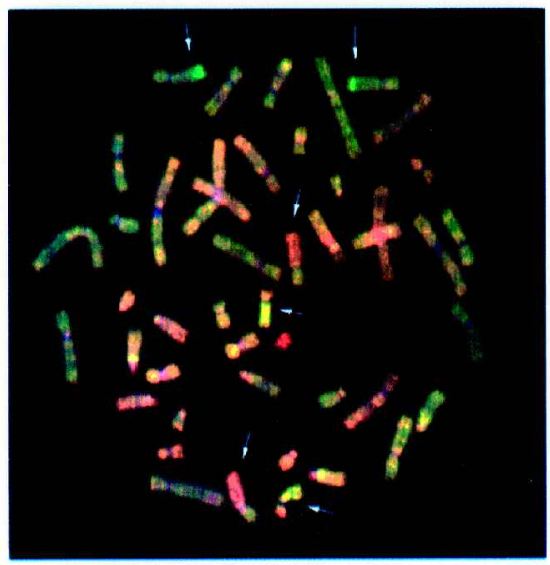

B
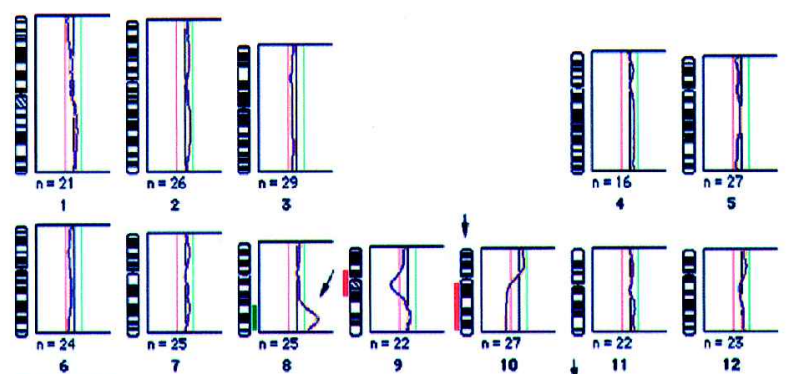

1
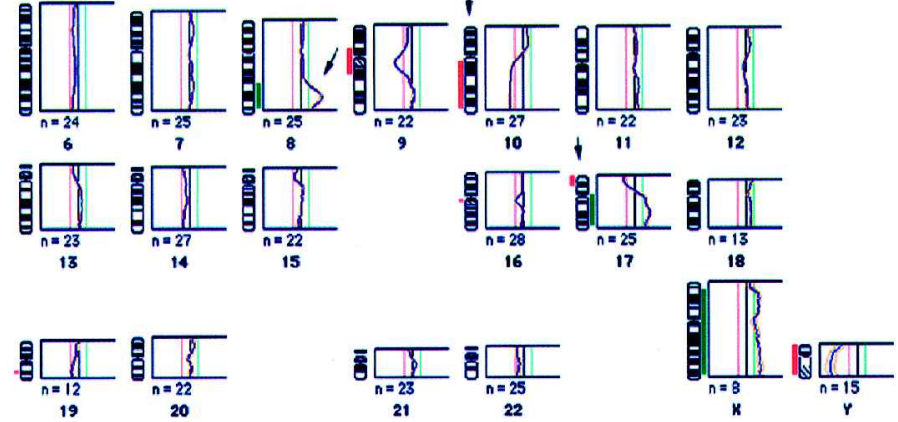

Fig. 2. CGH of D-425. A. Metaphase image. Arrows point to chromosomes with detectable numeric genomic changes. B. Ratio profile. The CGH data show amplification of chromosome region 8q22 $\rightarrow$ qter, which is inclusive of the locus of MYC (green), the deletion of 10q (red), and the deletion of the distal portion of $17 p$ in red and gain of $17 q$ in green.

G-banded chromosome spreads examined showed dmins, confirmed as containing gene amplification by using a FISH probe specific for the MYC gene in chromosome region 8q24.1 (Fig. 1B). These data correlated with the CGH findings, which showed amplification of the same region. Chromosome 10 had a terminal deletion of the long arm with a breakpoint at 10q23 according to cytogenetics. This observation was confirmed by a positive signal with a whole chromosome 10 paint (Fig. 1B). However, there was a lack of signal for a telomeric paint specific for the 10q26 region (data not shown). The CGH profile fits well with this finding, confirming the 10q deletion (Fig. 2). $\mathrm{CGH}$ also demonstrated a gain of $17 \mathrm{q}$ and terminal deletion of $17 \mathrm{p}$ (Fig. 2), correlating well with abnormal chromosome 17 seen in the karyotype (Fig. 1). The $\mathrm{i}(17 \mathrm{q}$ ) chromosome apparent by G-banding was shown to be a $\mathfrak{t}(17 ; 17)(\mathrm{p} 12 ; \mathrm{q} 11.2)$ chromosome by FISH (Table 1) (Aldosari et al., 2000). The SKY analysis was performed on metaphases from an in vitro passage that was later than those studied in the G-banded and FISH analyses. This preparation was hyperploid and showed an additional chromosomal abnormality, $\operatorname{der}(3 ; 10) \mathrm{t}(10 ; 3 ; 8)$.
Like G-banding, SKY identified the abnormal 17 chromosome as an $\mathrm{i}(17 \mathrm{q})$, and similar to FISH, the dmins were shown to be composed of chromosome 8 DNA sequences.

\section{D-556}

By conventional karyotyping, this cell line had a diploid chromosome number and contained few chromosomal abnormalities, which included abnormalities of chromosomes 1 and 17 and dmins (Table 1 and Fig. 3). Chromosome 13 appeared to be involved in a translocation with 1q. Whole chromosome paints and centromere paints for chromosomes 1 and 13 confirmed this unbalanced translocation of chromosomes 1 and 13 with breakpoints at $1 \mathrm{p} 11.1$ and $13 \mathrm{q} 34$. The derivative chromosome 13 was dicentric, containing chromosome 1 and 13 centromeres. This was confirmed by SKY (Fig. 3C). The CGH result suggested an extra copy of $1 \mathrm{q}$ and no changes in chromosome 13, in agreement with the cytogenetic, FISH, and SKY findings (Figs. 3 and 4). MYC amplification was demonstrated in all cells examined by using a gene-specific $\mathrm{LSI}^{\circledR}$ c-myc probe (Figs. 3B and 5). 


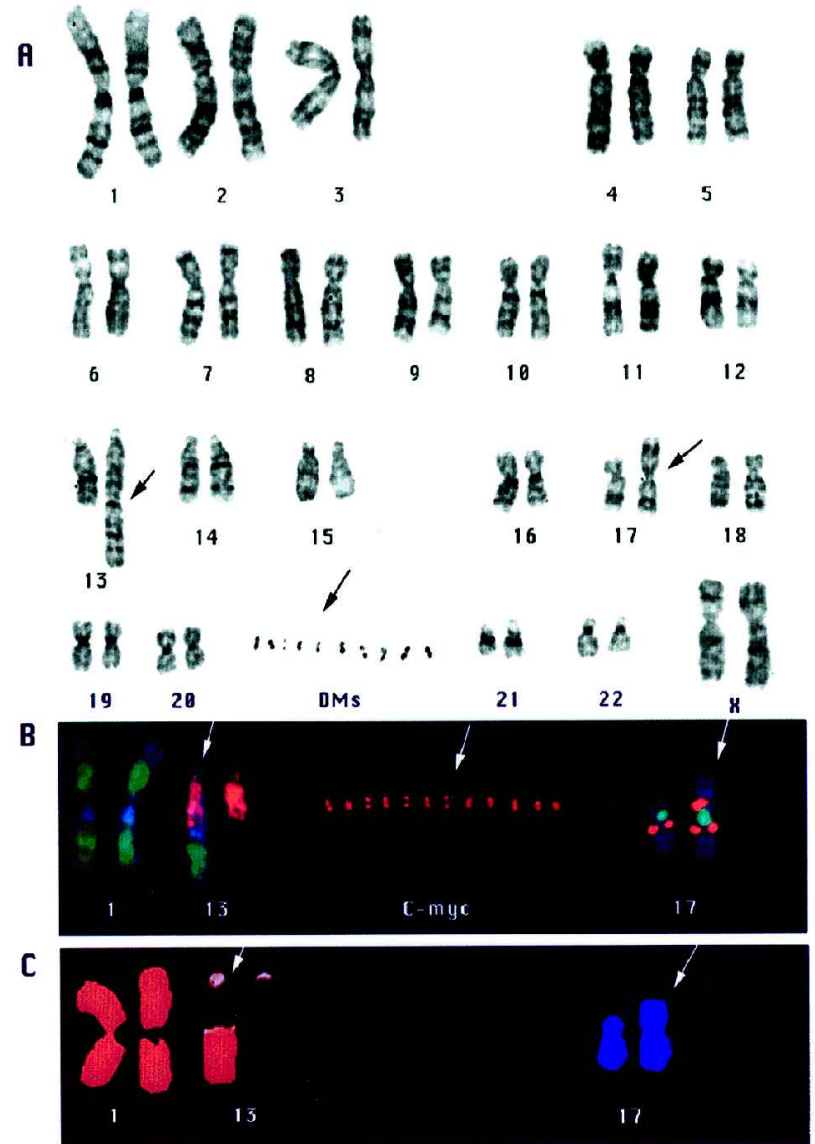

Fig. 3. Cell line D-556. A. Representative karyotype: 46,XX,+1, dic (1;13)(p11.1;q34),i(17)(q10), +dmins Arrows point to abnormal chromosomes and dmins. B. Composite panel showing results of FISH on normal and derivative chromosomes. Arrows indicate dic $(1 ; 13)$ chromosome (WCP 1 SpectrumGreen and WCP 13 SpectrumOrange), C-myc amplification in dmins (LSI c-myc SpectrumOrange), and the apparent isodicentric chromosome 17q (centromeric 17 SpectrumGreen and HER2 SpectrumOrange, 17q12). C. Composite panel showing SKY results for chromosomes $1,13,17$, and $\operatorname{dic}(1 ; 13)$, and the apparent $i(17 q)$. Arrows point to derivative chromosomes.

This finding was confirmed by CGH, but not by SKY. The $\mathrm{i}(17 \mathrm{q})$ chromosome identified in the karyotypes was shown by SKY to be composed entirely of chromosome 17 and by the initial FISH analysis to be composed of the $17 \mathrm{q}$ chromosome arm (Fig. 3A-3C). However, an idic $(17 \mathrm{q})$ chromosome was demonstrated by subsequent FISH analyses (Table 1) (Aldosari et al., 2000). This finding was seen as a gain of $17 \mathrm{q}$ and loss of the distal portion of $17 \mathrm{p}$ by CGH (Fig. 4).

\section{D-721}

This cell line had a near-diploid chromosome number and contained a wide range of complex chromosomal rearrangements. By conventional karyotyping, it was not possible to determine all of the partner chromosomes involved in translocations or the identity of all marker chromosomes (Fig. 6). FISH was instrumental in fully describing the translocations $\operatorname{der}(1) t(1 ; 18), \operatorname{dic}(3 ; 22)$, and $\operatorname{der}(18)(18 ; 20)$; refining the breakpoints for chromosomes $\operatorname{der}(11) \mathrm{t}(3 ; 11)$ and $\operatorname{dic}(16 ; 22)$; and confirming the $\operatorname{dic}(1 ; 9)$ chromosome (Table 1$)$. The SKY results confirmed the majority of these findings and provided evidence suggesting that the add(18) chromosome identified by G-banding was a duplication of chromosome 18 (data not shown). WCP for chromosome 18 revealed that the marker chromosome was made up solely of chromosome 18 material (Table 1). The CGH results corroborated with the G-band, FISH, and SKY results, showing increased copy numbers of $1 \mathrm{q}, 3 \mathrm{p}, 9$; amplification of $18 \mathrm{q}$; and decreased copy numbers of $\mathrm{Y}, 1 \mathrm{pter}->\mathrm{p} 32.2,3 \mathrm{q}$, 11p15, 16q, 20p, and 22 loci.

\section{D-690}

Karyotypic analysis showed this xenograft as having a diploid chromosome number and containing markers and complex chromosomal rearrangements involving most of the chromosomes. Interestingly, despite the complexity of the chromosomal pattern, this cell line contained many simple balanced reciprocal translocations, as well as some complex chromosomal rearrangements that could be fully appreciated only by subsequent FISH and SKY analyses. The initial composite karyotype, 46,Y, $\operatorname{der}(\mathrm{X}) \mathrm{t}(\mathrm{X} ; 7)(\mathrm{p} 11.2 ; \mathrm{p} 13), \operatorname{der}(2) \mathrm{t}(2 ; 4)(\mathrm{p} 13 ; \mathrm{p} 14) \mathrm{t}(2 ; 11)(\mathrm{q} 37 ;$ $\mathrm{q} 21), \operatorname{der}(4) \mathrm{t}(4 ; 12)(\mathrm{p} 14 ; \mathrm{q} 12), \operatorname{der}(6) \mathrm{t}(6 ; ?)(\mathrm{q} 21 ; ?),-7, \mathrm{t}(8 ; 15)$ (q22;q22)[2],der(10)t(10;?)(q22;?),del(11)(q21),der(11)t $(11 ; 12)(\mathrm{p} 15 ; \mathrm{q} 22), \operatorname{der}(12) \mathrm{t}(12 ; ?)(\mathrm{q} 13 ; ?),-12$,ins(13;?)(q21;?), 
A

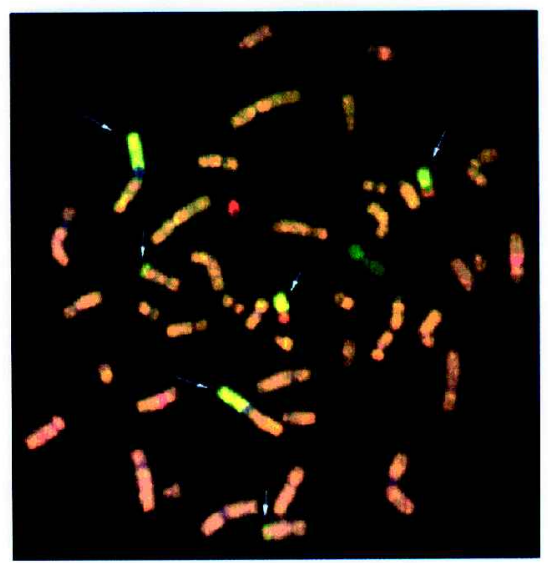

B

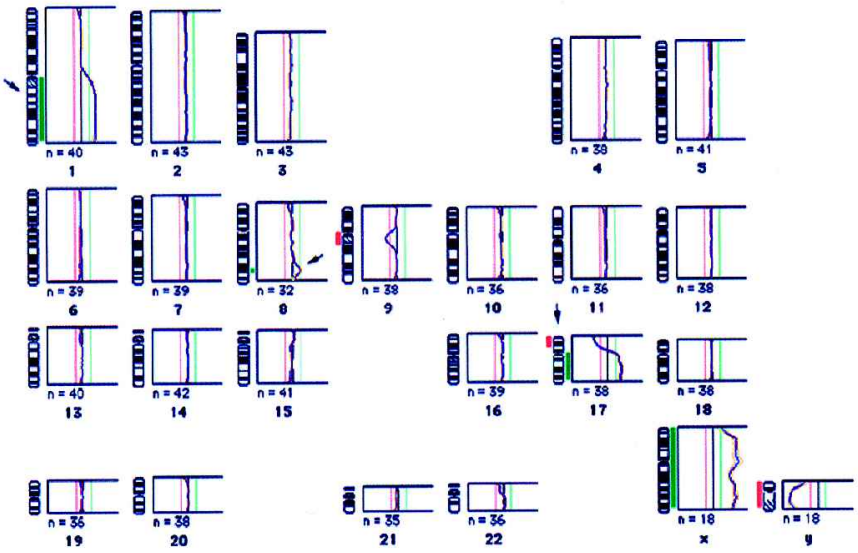

Fig. 4. CGH of D-556. A. Metaphase image. Arrows point to chromosomes with detectable numeric genomic changes. B. Ratio profile. The CGH data show gain of chromosome 1q, gain of chromosome region 8q23-8q24, deletion of 17p, and gain of $17 q$.

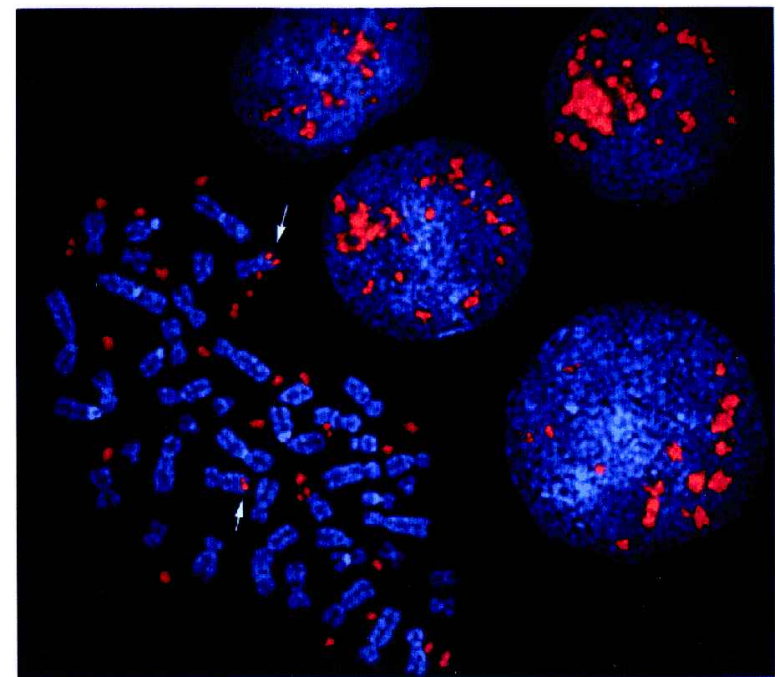

Fig. 5. Cell line D-556. FISH analysis shows a metaphase spread and interphase nuclei according to an LSI c-myc gene probe. Arrows indicate the locus of MYC on chromosome 8q24.1.

$\operatorname{der}(22) \mathrm{t}(7 ; 22)(\mathrm{q} 21 ; \mathrm{q} 13),+2 \mathrm{mar}[\mathrm{cp} 7]$, was reevaluated by FISH (Fig. 7). WCPs were important in correctly identifying $\operatorname{der}(2) \mathrm{t}(2 ; 4 ; 11)$, der(9)t(9;22), del(10)(q22q24), and ins $(13 ; 1)$, and in identifying the 2 marker chromosomes as $\operatorname{der}(7) \mathrm{t}(\mathrm{X} ; 2 ; 7)$ and the derivative chromosome 12 partner of the balanced translocation $(4 ; 12)$. Furthermore, it was easier to recognize the balanced translocations $\mathrm{t}(4 ; 12), \mathrm{t}(6 ; 20)$, and $\mathrm{t}(11 ; 12)$ and confirm $\operatorname{der}(\mathrm{X}) \mathrm{t}(\mathrm{X} ; 7)$, $\mathrm{t}(8 ; 15)$, and $\operatorname{der}(22) \mathrm{t}(7: 22)$ by FISH analysis (Table 1 and Fig. 7A and 7B). SKY confirmed our findings in this xenograft and detected rearrangements not easily discerned by either G-bands or FISH. The der(4) chromosome from the apparent balanced translocation $(4 ; 12)$ also was shown to be composed of a small part of chromosome 11 at the terminal end of the long arm of chromosome 4 , making the description $\operatorname{der}(4) \mathrm{t}(4 ; 12)(\mathrm{p} 15$; q13)t(4;11)(q35;?) (Fig. 7C). Furthermore, the del(11) chromosome was shown to be translocated with a part of chromosome 2 (Fig. 7C). Even though a great amount of complexity was seen in the karyotypes, the net change of DNA sequences seen by CGH analysis was simpler. It showed only a deletion of region 10q22-10q24, with all other chromosomes having no changes (Fig. 8).

\section{D-581}

The G-banded karyotype revealed numerous structural deviations (Fig. 9). FISH, used with WCPs, permitted the identification of the translocation partners in a number of unbalanced translocations. For instance, FISH helped to correctly identify $\operatorname{der}(2) \mathrm{t}(2 ; 3)$ as $\operatorname{dic}(2 ; 10) t(2 ; 10 ; 13)$; 


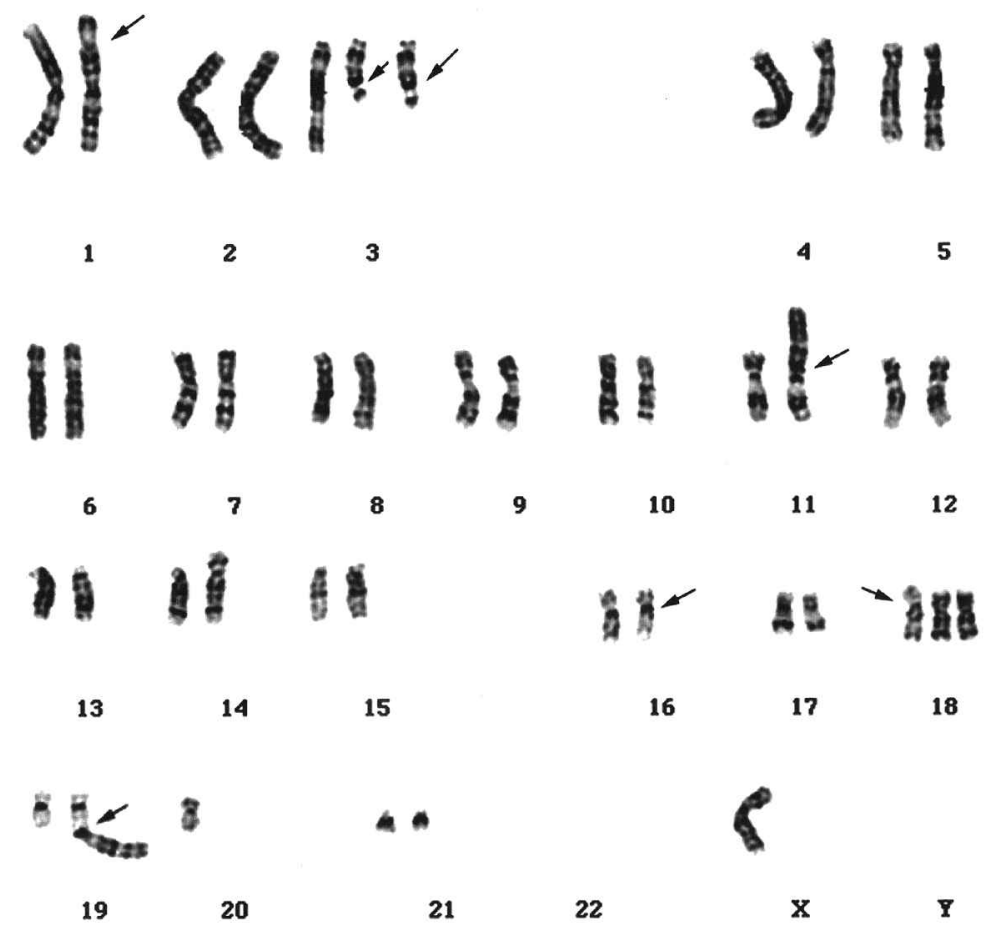

Fig. 6. Cell line D-721. Representative karyotype: 44,X,-Y,+der(1)t(1;?)(p34.1;?),der(3)t(3;?)(p10:?),+der(3)t(3;?)(p10:?),der(5)t(5;?)(q?;?),der $(11) t(3 ; 11)(q 12 ; p 15), \operatorname{add}(14)(p 11.2), \operatorname{dic}(16 ; 22)(q 12.1 ; p 11.1), a d(18)(p 11.3) \times 2,+\operatorname{der}(18) t(18 ; ?)(q 10 ; ?), \operatorname{dic}(1 ; 19)(p 11.1 ; q 13.4),-20,-22,-22$ Chromosomes der(5) and add(14) are unique to this cell and are therefore not clonal. Arrows indicate chromosomal breakpoints determined by G-bands and FISH analyses.

$\operatorname{der}(3) \mathrm{t}(3 ; 3)$ as $\operatorname{der}(3) \mathrm{t}(3 ; 13 ; 15)$; and $\operatorname{der}(5) \mathrm{t}(2 ; 5)$ as $\operatorname{der}(5) \mathrm{t}(4 ; 5)$ (Table 1). Rearranged chromosomes that were not entirely discerned by G-bands were fully characterized by FISH, including $\operatorname{der}(1) \mathrm{t}(1 ; 10)$, der(9)t(1;9), $\operatorname{der}(10) t(2 ; 10 ; 13)$, der(10)t $(9 ; 10)$, and $\operatorname{der}(13) t(13 ; 22)$ (Table 1). The marker chromosome was shown to be composed entirely of chromosome 15, whereas $\operatorname{der}(2) \mathrm{t}(1 ; 2)$ and del(17) were confirmed (Table 1). Due to technical limitations, SKY data was not obtained on this cell line. $\mathrm{CGH}$ also revealed gains and losses involving almost all the chromosomes in this cell line, which was consistent with the karyotypic and FISH data. Gains of $1 \mathrm{q}, 2,3 \mathrm{q}, 4 \mathrm{q}, 7,13$, and 15 , along with losses on Y, 5p, $10,17 \mathrm{p}$, and 20 were observed.

\section{Discussion}

Cultured cell lines and xenografts derived from human neoplasms are useful for a variety of investigational studies, including delineation of molecular alterations that contribute to the biologic properties of the neoplasm (Friedman et al., 1983). Understanding of molecular characteristics is aided by elucidation of chromosomal alterations that a given tumor type possesses.

Conventional cytogenetics using G-banded karyotypes is a well-known method that in the last few decades has been used to define numerical and structural alterations, including translocations, deletions, and inversions in the chromosomes of human tumors (Bigner and Vogelstein, 1990). For some tumors, the metaphases are easy to ana- lyze with this method because there are few altered chromosomes (Lopez-Gines et al., 1997; Stratton et al., 1991). In other cases, complex karyotypes with numerous chromosomal rearrangements and marker chromosomes are seen, making precise identification of all structural and numerical changes difficult or impossible (Bigner et al., 1988, 1997; Grant et al., 1988; Keles et al., 1995; Sawyer et al., 1991). One cell line in the current study (D-556) had very simple chromosomal alterations consisting of a translocation (1:13), isochromosome $(17 \mathrm{q})$, and dmins. The other cell lines and xenograft showed more complex chromosomal rearrangements that could not be analyzed by this method alone.

Major advances in molecular cytogenetics in the last decade have provided new methods such as FISH, SKY, and $\mathrm{CGH}$, which have allowed continued analysis of chromosomes beyond G-bands. FISH includes specific chromosome-painting probes, centromeric probes, telomeric probes, specific locus probes, and specific gene probes (Adinolfi and Davies, 1994). This method enables investigators to evaluate gains and losses of chromosomes and chromosomal regions and to analyze complex rearrangements within individual cells (Tomlinson et al., 1994; Vagner-Capodano et al., 1994). It is also useful for chromosomal mapping and is especially powerful when applied along with loss of heterozygosity studies (Aldosari et al., 2000; McDonald et al., 1994; SteichenGersdorf et al., 1997). In this study, we used a MYC gene-specific probe in cell lines D-556 and D-425 to demonstrate MYC gene amplification in dmins (Bigner et al., 1990). Using multicolor painting probes, we were 


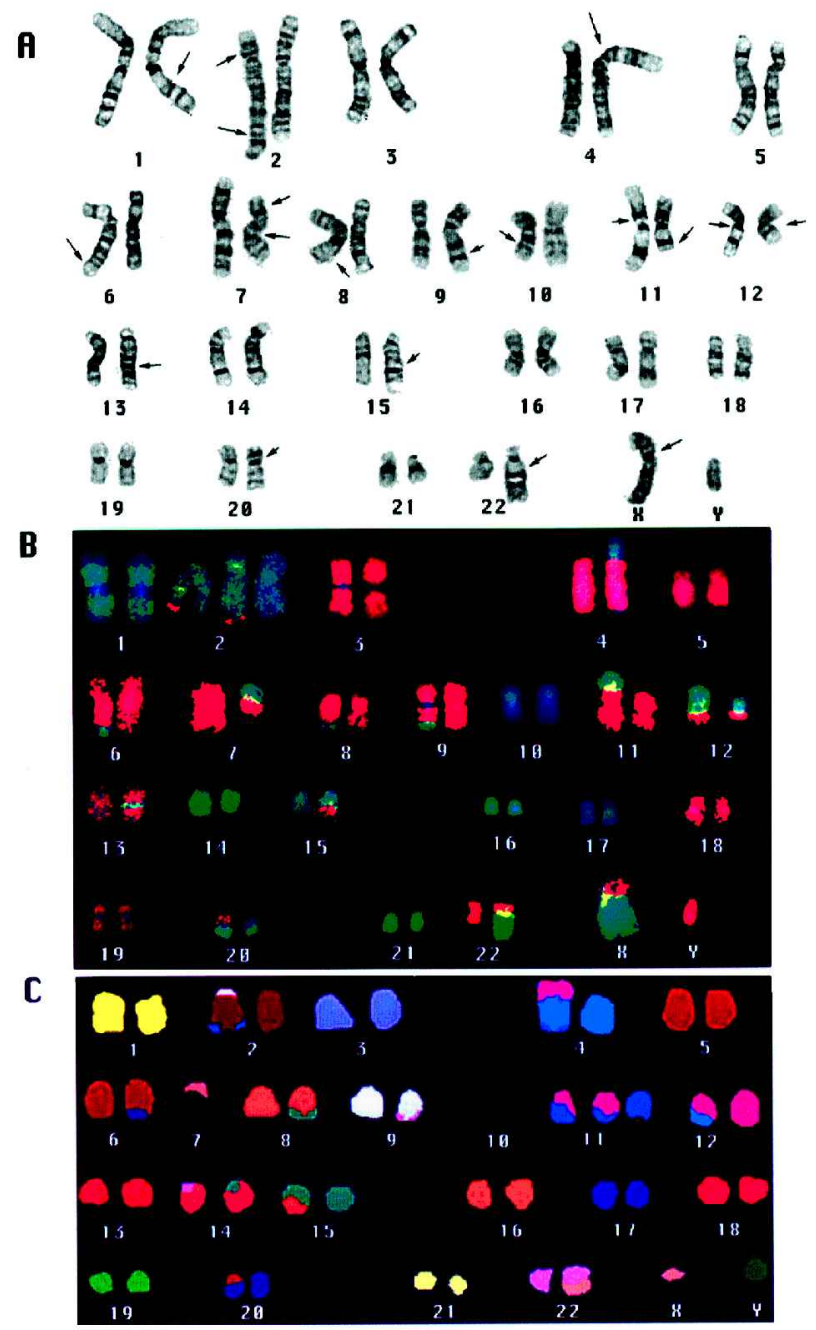

Fig. 7. Xenograft D-690. A. Representative G-banded karyotype with arrows pointing to breakpoints of the abnormal chromosomes determined by G-bands and FISH. B. Composite panel showing results of FISH. Each abnormality was investigated with a specific combination of probes (Table 1). C. Composite panel showing results of SKY for a representative metaphase spread.

able to describe most chromosomal rearrangements in all 5 tumors. Specifically, we confirmed the simple translocation in D-556 and delineated complex rearrangements in D-690, D-581, and D-721. The advantage of this method is that one can precisely confirm chromosomal abnormalities that involve a limited number of chromosomes. The disadvantage of this method is that it requires metaphase spreads, and it is time consuming and costly to analyze cell lines or xenografts that have multiple rearrangements which involve most of their chromosomes. In this setting, a simpler approach is to perform SKY.

SKY was introduced to the field of molecular cytogenetics in the last few years. In this method, combinations of 6 fluorochromes are used to paint the different chromosomes. Each pair of chromosomes is assigned to a pseudocolor using computer software and a fluorescent microscope (Schrock et al., 1996). This method is partially useful in cases with very complex chromosomal rearrangements that involve most or all of the chromosomes (Bigner and Schrock, 1997). In medulloblastomas, karyotypes vary from simple patterns, such as in D-425 and D-556, to complex karyotypes with multiple chromosomal rearrangements that involve most of the chromosomes. Application of SKY to xenograft D-690 demonstrated the ability of this method to define subtle rearrangements involving chromosome 2 and complex rearrangements involving 3 chromosomes, der(4)t(4;12;11). FISH showed only the translocation between chromosomes 4 and 12 , failing to show the other partner chromosomes. The major drawbacks of this method, however, are that it requires metaphase spreads and cannot show deletions smaller than 2 megabases.

CGH allows one to investigate the net gains, losses, and amplification of chromosomes and chromosomal regions using DNA extracted from tumor. Unlike loss of heterozygosity studies, normal DNA from the individual is not needed, and unlike conventional cytogenetics, FISH and SKY, metaphase spreads are not required. For CGH, DNA from normal control cells is labeled with a red fluorochrome, and tumor DNA is labeled with green (Kallioniemi et al., 1992). The samples are mixed in equal quantities and hybridized to normal metaphase spreads 

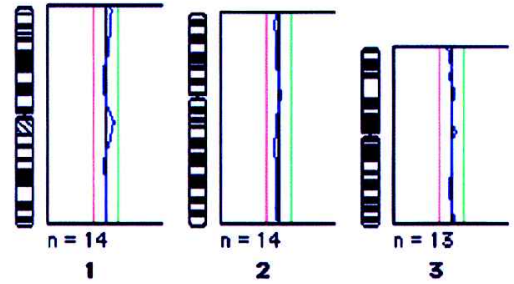

D-690
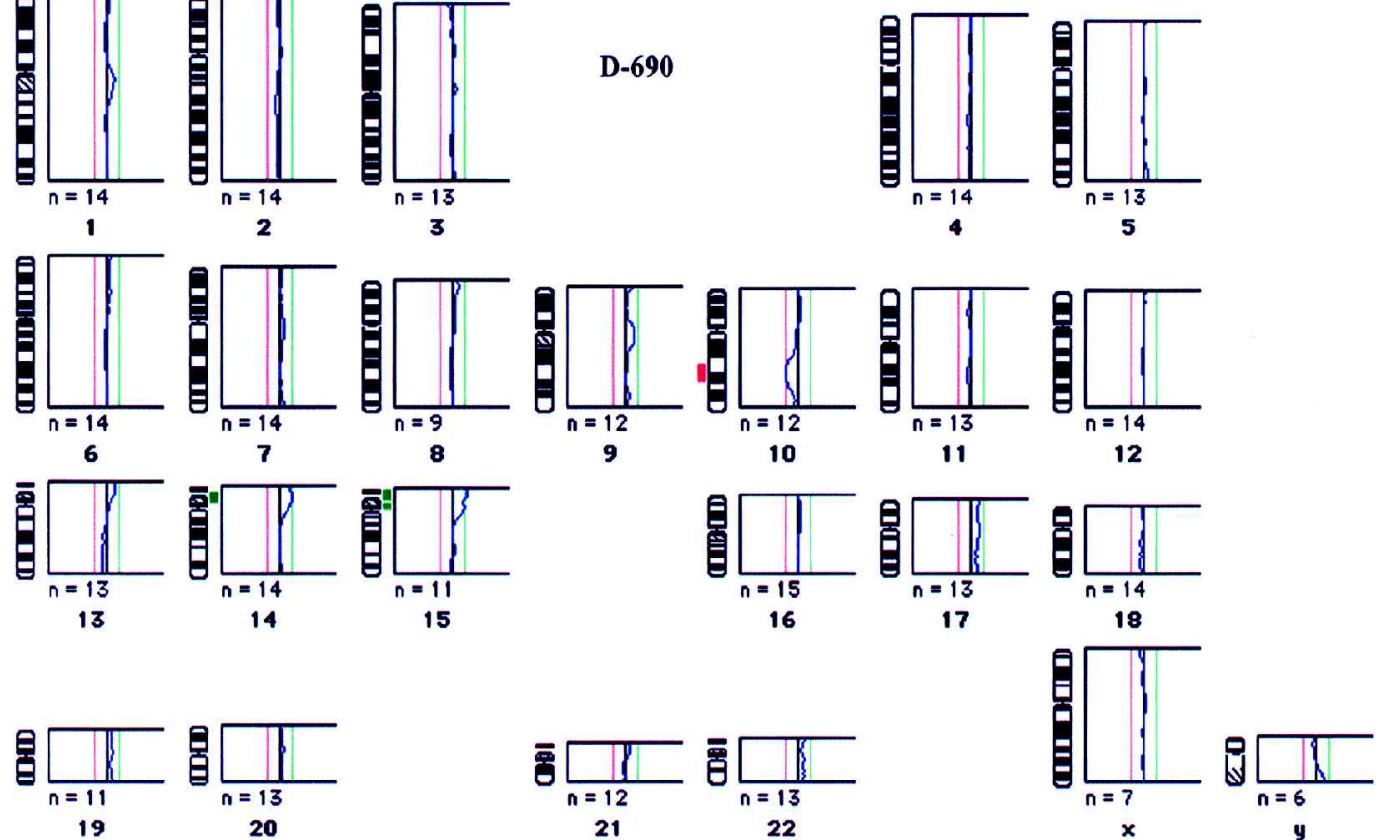

Fig. 8. CGH ratio profile. The $\mathrm{CGH}$ data show deletion of chromosome 10q, region 10q22-10q24, as the only abnormality seen in this xenograft.
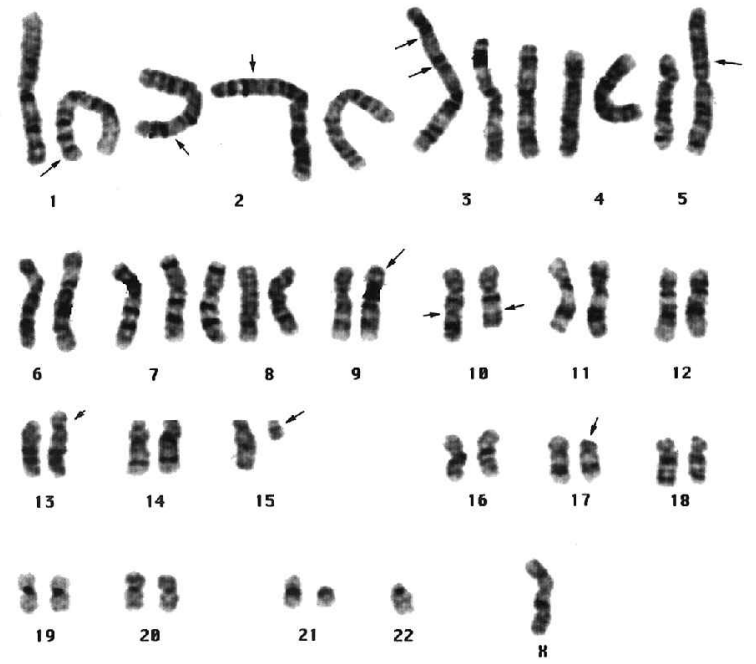

Fig. 9. Cell line D-581. Initial G-banded karyotype: $47, X,-Y, \operatorname{add}(1)(q 44), \operatorname{der}(2) t(1 ; 2)(q 21 ; p 23),+\operatorname{der}(2) t(2 ; 3)(q 23 ; q 23)$, $+\operatorname{der}(3) \mathrm{t}(3: 3)(\mathrm{p} 25 ; \mathrm{q} 21), \operatorname{der}(5) \mathrm{t}(2 ; 5)(\mathrm{q} 24 ; \mathrm{p} 15.1),+7, \operatorname{add}(9)(\mathrm{p} 2), \operatorname{der}$ (10)t(10:?)(q22;?), add(10)(q23.2), add(13)(p11),-15, del(17)(p12),$22,+$ mar. Arrows indicate chromosomal breakpoints and marker chromosomes determined by subsequent FISH analysis (Table 1).

from a normal individual. This method is particularly suited to demonstrating gene amplification (Jay et al., 1999). It is useful for providing a complete chromosomal pattern (Reardon et al., 1997; Thein et al., 2000). In the present series, chromosomal gains, losses, and MYC amplification were all confirmed. In addition, CGH demonstrates that the complex pattern of chromosomal rearrangements in xenograft D-690 Med actually resulted in only a minimal alteration of the overall chromosomal complement, consisting of loss of 10q22-q24.
The combinatorial analysis presented in this study provides some insight into the genetic alterations of these 4 medulloblastoma cell lines and xenograft. Alterations were detected with all chromosomes except chromosomes 14 and 21. Of these, chromosome 1 was involved in the most alterations, detected in 4 of the 5 samples, followed by chromosomes $8,9,10,13,17$, and 22, each detected in 3 of 5 samples (Table 1). Alterations involving the remaining chromosomes were observed in only 1 or 2 of the specimens.

Isochromosome $17 \mathrm{q}$ has been reported in molecular and cytogenetic studies as the most common aberration identified in medulloblastoma cell lines and biopsies (Aldosari et al., 2000; Bigner et al., 1997). The rearrangement results in loss of $17 \mathrm{p}$ and gain of $17 \mathrm{q}$. Our study agrees with the published data, but shows that the common alteration may not always be a simple isochromosome. The $\mathrm{i}(17 \mathrm{q})$ identified in $\mathrm{D}-425$ and D-556 cell lines has been elucidated as $t(17 ; 17)$ and idic(17), respectively, with the aid of molecular cytogenetic techniques (Table 1) (Aldosari et al., 2000). Chromosome 1 has been involved in various structural alterations in primary medulloblastoma, which resulted in a gain of 1q without loss of 1p (Bigner et al., 1997). This was also consistent in this study with a net gain of chromosome 1q detected in samples D-556, D-581, and D-721. Interestingly, in the xenograft D-690, the analyses suggest that $1 \mathrm{q} 25 \rightarrow \mathrm{q} 31$ was inserted into the long arm of chromosome 13, making it the smallest region of $1 \mathrm{q}$ to be involved in a rearrangement. Presently, it is not clear if this loci is simply gained or involved in a balanced rearrangement. Additional investigation of this insertion would determine its potential as a putative oncogenic loci in medulloblastoma. Loss of chromosome $10 \mathrm{q}$ in medulloblastoma is another frequently reported alteration that has also been detected in our study (Rear- 
don et al., 1997). The combinatorial approach on these cell lines and xenograft pinpointed a cluster of breakpoints around chromosome locus $10 \mathrm{q} 23$ in D-425, D-581, and D-690. Although the PTEN gene, located in this region, has been mutated in a few medulloblastomas, it has not been implicated in the development or maintenance of the tumor (Giangaspero et al., 2000). Although no other candidate tumor suppressor genes have been identified in $10 \mathrm{q} 23$, the data from this investigation, along with other published studies, maintain that this locus is potentially important in the etiology of medulloblastoma. Amplification of the MYC gene, on $8 \mathrm{q} 24$, is seen in medulloblastoma biopsies and cell lines (Bigner and Vogelstein, 1990). This is the most common documented alteration involving chromosome 8 in this tumor, which was also seen in this study. Two of the 3 alterations involving chromosome 8 were MYC amplification, in the form of dmins, in cases D-425 and D-556. Alterations involving chromosomes 9, 13, and 22 have not been frequently reported; thus, the alterations seen in this study are probably unique to these medulloblastoma samples.

Combining classic cytogenetics and molecular cytogenetics was clearly beneficial in discerning the complex genetic nature of medulloblastoma cell lines and xenografts. The study provided, for the first time, detailed genetic analysis of medulloblastoma samples, the results of which can be used in other investigations. Furthermore the data, corroborated by published reports, indicated the necessity for reevaluating an apparent common chromosome alteration and suggested a potential oncogenic locus that is possibly important in the etiology of medulloblastoma.

\section{Acknowledgments}

The authors acknowledge the technical assistant of Linda Cleveland, Jan Campbell, and Nancy Bullock.

\section{References}

Adinolfi, M., and Davies, A.F. (1994) Non-Isotopic In Situ Hybridization: Applications to Clinical Diagnosis and Molecular Genetics. Austin: R.G. Landes.

Aldosari, N., Rasheed, B.K.A., McLendon, R.E., Friedman, H.S., Bigner, D.D., and Bigner, S.H. (2000) Characterization of chromosome 17 abnormalities in medulloblastomas. Acta Neuropathol. 99, 345-351.

Bigner, S.H., and Schrock, E. (1997) Molecular cytogenetics of brain tumors. J. Neuropathol. Exp. Neurol. 56, 1173-1181.

Bigner, S.H., and Vogelstein, B. (1990) Cytogenetics and molecular genetics of malignant gliomas and medulloblastoma. Brain Pathol. 1, 12-18.

Bigner, S.H., Mark, J., Friedman, H.S., Biegel, J.A., and Bigner, D.D. (1988) Structural chromosomal abnormalities in human medulloblastoma. Cancer Genet. Cytogenet. 30, 91-101.

Bigner, S.H., Friedman, H.S., Vogelstein, B., Oakes, W.J., and Bigner, D.D. (1990) Amplification of the c-myc gene in human medulloblastoma cell lines and xenografts. Cancer Res. 50, 2347-2350.

Bigner, S.H., McLendon, R.E., Fuchs, H., McKeever, P.E., and Friedman, H.S. (1997) Chromosomal characteristics of childhood brain tumors. Cancer Genet. Cytogenet. 97, 125-134.

Friedman, H.S., Bigner, S.H., McComb, R.D., Schold, S.C., Jr., Pasternak, J.F., Groothuis, D.R., and Bigner, D.D. (1983) A model for human medulloblastoma: Growth, morphology, and chromosomal analysis in vitro and in athymic mice. J. Neuropathol. Exp. Neurol. 42, 485-503.

Giangaspero, F., Bigner, S.H., Kleihues, P., Pietsch, T., and Trojanowski, J.Q. (2000) Medulloblastoma. In: Kleihues, P., and Cavenee,W.K. (Eds.), Pathology and Genetics of Tumours of the Nervous System: World Health Organization Classification of Tumors. Lyon, France: IARC Press. pp. 129-137.

Gozzetti, A., and Le Beau, M.M. (2000) Fluorescence in situ hybridization: Uses and limitations. Semin. Hematol. 37, 320-333.

Grant, G., Pathak, S., and Maria, B.L. (1988) Identification of marker chromosomes in a human medulloblastoma cell line (D283 Med). Cancer Genet. Cytogenet. 34, 247-250

Jay, V., Squire, J., Bayani, J., Alkhani, A.M., Rutka, J.T., and Zielenska, M. (1999) Oncogene amplification in medulloblastoma: Analysis of a case by comparative genomic hybridization and fluorescence in situ hybridization. Pathology 31, 337-344.

Kallioniemi, A., Kallioniemi, O.P., Sudar, D., Rutovitz, D., Gray, J.W., Waldman, F., and Pinkel, D. (1992) Comparative genomic hybridization for molecular cytogenetic analysis of solid tumors. Science 258, 818-821.

Keles, G.E., Berger, M.S., Srinivasan, J., Kolstoe, D.D., Bobola, M.S., and Silber,
J.R. (1995) Establishment and characterization of four human medulloblastoma-derived cell lines. Oncol. Res. 7, 493-503.

Lichter, P., Joos, S., Bentz, M., and Lampel, S. (2000) Comparative genomic hybridization: Uses and limitations. Semin. Hematol. 37, 348-357.

Lopez-Gines, C., Cerda-Nicolas, M., Gil-Benso, R., Barcia-Salorio, J.L., and Llombart-Bosch, A. (1997) Involvement of the long arm of chromosome 9 in medulloblastoma in an adult. Cancer Genet. Cytogenet. 96, 81-84.

McDonald, J.D., Daneshvar, L., Willert, J.R., Matsumura, K., Waldman, F., and Cogen, P.H. (1994) Physical mapping of chromosome 17p13.3 in the region of a putative tumor suppressor gene important in medulloblastoma. Genomics 23, 229-232.

Mitelman, F. (Ed.) (1995) ISCN 1995: An International System for Human Cytogenetic Nomenclature. Basel, Switzerland: S. Karger.

Reardon, D.A., Michalkiewicz, E., Boyett, J.M., Sublett, J.E., Entrekin, R.E., Ragsdale, S.T., Valentine, M.B., Behm, F.G., Li, H., Heideman, R.L., Kun, L.E., Shapiro, D.N., and Look, A.T. (1997) Extensive genomic abnormalities in childhood medulloblastoma by comparative genomic hybridization. Cancer Res. 57, 4042-4047.

Sawyer, J.R., Swanson, C.M., Roloson, G.J., Longee, D.C., Boop, F.A., and Chadduck, W.M. (1991) Molecular cytogenetic analysis of a medulloblastoma with isochromosome 17 and double minutes. Cancer Genet. Cytogenet. 57, 181-186.

Schrock, E., du Manoir, S., Veldman, T., Schoell, B., Wienberg, J., FergusonSmith, M.A., Ning, Y., Ledbetter, D.H., Bar-Am, I., Soenksen, D., Garini, Y., and Ried, T. (1996) Multicolor spectral karyotyping of human chromosomes. Science 273, 494-497.

Schrock, E., and Padilla-Nash, H. (2000) Spectral karyotyping and multicolor fluorescence in situ hybridization reveal new tumor-specific chromosomal aberrations. Semin. Hematol. 37, 334-347.

Steichen-Gersdorf, E., Baumgartner, M., Kreczy, A., Maier, H., and Fink, F.M. (1997) Deletion mapping on chromosome 17p in medulloblastoma. Br. J. Cancer 76, 1284-1287.

Stratton, M.R., Darling, J., Cooper, C.S., and Reeves, B.R. (1991) A case of cerebellar medulloblastoma with a single chromosome abnormality. Cancer Genet. Cytogenet. 53, 101-103.

Thein, A., Trkova, M., Fox, M., and Parrington, J. (2000) The application of comparative genomic hybridization to previously karyotyped cervical cancer cell lines. Cancer Genet. Cytogenet. 116, 59-65.

Tomlinson, F.H., Jenkins, R.B., Scheithauer, B.W., Keelan, P.A., Ritland, S., 
Parisi, J.E., Cunningham, J., and Olsen, K.D. (1994) Aggressive medulloblastoma with high-level N-myc amplification. Mayo Clinic Proc. 69, 359-365.

Vagner-Capodano, A.M., Zattara-Cannoni, H., Gambarelli, D., Gentet, J.C., Genitori, L., Lena, G., Graziani, N., Raybaud, C., Choux, M., and Grisoli, F. (1994) Detection of $\mathrm{i}(17 \mathrm{q})$ chromosome by fluorescent in situ hybridization (FISH) with interphase nuclei in medulloblastoma. Cancer Genet. Cytogenet. 78, 1-6.
Wiltshire, R.N., Rasheed, B.K.A., Friedman, H.S., Friedman, A.H., and Bigner, S.H. (2000) Comparative genetic patterns of glioblastoma multiforme: Potential diagnostic tool for tumor classification. Neuro-Oncology [serial online], Doc. 00-015, June 20, 2000. URL http://neuro-oncology. mc.duke.edu. Neuro-Oncology 2, 164-173.

Wiltshire, R.N., Dennis, T.R., Sondak, V.K., Meltzer, P.S., and Trent, J.M. (2001) Application of molecular cytogenetic techniques in a case study of human cutaneous metastatic melanoma. Cancer Genet. Cytogenet. 131, 97-103. 